\title{
Uncertainty and sensitivity analysis of the basic reproduction number of diphtheria: A case study of Rohingya refugee camp in Bangladesh, November-December 2017
}

\author{
Ryota Matsuyama ${ }^{1,2}$, Andrei R Akhmetzhanov ${ }^{1,2}$, Akira Endo ${ }^{1,3}$, Hyojung Lee ${ }^{1,2}$, Takayuki Yamaguchi ${ }^{1,2}$, \\ Shinya Tsuzuki ${ }^{1,2}$, Hiroshi Nishiura ${ }^{\text {Corresp. } 1,2}$ \\ ${ }^{1}$ Graduate School of Medicine, Hokkaido University, Sapporo, Japan \\ 2 Japan Science and Technology Agency, Core Research for Evolutional Science and Technology, Saitama, Japan \\ 3 London School of Hygiene \& Tropical Medicine, University of London, London, United Kingdom \\ Corresponding Author: Hiroshi Nishiura \\ Email address: nishiurah@gmail.com
}

Background. A Rohingya refugee camp in Cox's Bazar, Bangladesh experienced a largescale diphtheria epidemic in 2017 . The background information of previously immune fraction among refugees cannot be explicitly estimated, and thus, we conducted an uncertainty analysis of the basic reproduction number, R0. Methods. A renewal process model was devised to estimate the R0 and ascertainment rate of cases, and loss of susceptible individuals was modeled as one minus the sum of initially immune fraction and the fraction naturally infected during the epidemic. To account for the uncertainty of initially immune fraction, we employed a Latin Hypercube sampling (LHS) method. Results. R0 ranged from 4.7 to 14.8 with the median estimate at 7.2. R0 was positively correlated with ascertainment rates. Sensitivity analysis indicated that R0 would become smaller with greater variance of the generation time. Discussion. Estimated R0 was broadly consistent with published estimate from endemic data, indicating that the vaccination coverage of $86 \%$ has to be satisfied to prevent the epidemic by means of mass vaccination. LHS was particularly useful in the setting of refugee camp in which the background health status is poorly quantified. 


\section{Uncertainty and sensitivity analysis of the basic reproduction}

2 number of diphtheria: A case study of Rohingya refugee

3 camp in Bangladesh, November-December 2017

5 Ryota Matsuyama ${ }^{1,2}$, Andrei R. Akhmetzhanov ${ }^{1,2}$, Akira Endo ${ }^{1,3}$, Hyojung Lee ${ }^{1,2}$, Takayuki

6 Yamaguchi ${ }^{1,2}$, Shinya Tsuzuki ${ }^{1,2}$, Hiroshi Nishiura ${ }^{1,2 \S}$

$8{ }^{1 .}$ Graduate School of Medicine, Hokkaido University, Sapporo, Japan

9 2. CREST, Japan Science and Technology Agency, Saitama, Japan

10 3. London School of Hygiene and Tropical Medicine, London, United Kingdom

12 Corresponding Author:

13 Hiroshi Nishiura

14 Graduate School of Medicine, Hokkaido University, Sapporo, Japan

15 Kita 15 Jo Nishi 7 Chome, Kita-ku, Sapporo-shi, Hokkaido 060-8638, Japan

16 Email address: nishiurah@med.hokudai.ac.jp 


\section{Abstract}

\section{Background}

20 A Rohingya refugee camp in Cox's Bazar, Bangladesh experienced a large-scale diphtheria

21 epidemic in 2017. The background information of previously immune fraction among refugees

22 cannot be explicitly estimated, and thus, we conducted an uncertainty analysis of the basic

23 reproduction number, $R_{0}$.

\section{Methods}

25 A renewal process model was devised to estimate the $R_{0}$ and ascertainment rate of cases, and loss

26 of susceptible individuals was modeled as one minus the sum of initially immune fraction and

27 the fraction naturally infected during the epidemic. To account for the uncertainty of initially

28 immune fraction, we employed a Latin Hypercube sampling (LHS) method.

\section{Results}

$R_{0}$ ranged from 4.7 to 14.8 with the median estimate at 7.2. $R_{0}$ was positively correlated with

31 ascertainment rates. Sensitivity analysis indicated that $R_{0}$ would become smaller with greater

32 variance of the generation time.

\section{Discussion}

34 Estimated $R_{0}$ was broadly consistent with published estimate from endemic data, indicating that the vaccination coverage of $86 \%$ has to be satisfied to prevent the epidemic by means of mass vaccination. LHS was particularly useful in the setting of refugee camp in which the background

37 health status is poorly quantified. 


\section{Introduction}

40 Diphtheria, a bacterial disease caused by Corynebacterium diphtheriae, is a vaccine-preventable

41 disease. Symptomatic patients initially complain sore throat and fever. Additionally, a grey or

42 white patch causes the "croup", blocking the airway and causing a barking cough. Due to

43 widespread use of Diphtheria-Tetanus-Pertussis (DTP) vaccine globally, the incidence has

44 steadily declined over time, and thus, diphtheria is commonly perceived as almost a disease of

45 pre-vaccination era. Nevertheless, sporadic cases and even epidemics of the disease have been

46 yet reported especially in politically unstable areas, and many cases have been considered as

47 arising from susceptible pockets of the vulnerable population (Rusmil et al., 2015; Hosseinpoor

48 et al., 2016; Sangal et al., 2017).

In 2017, multiple diphtheria outbreaks were reported in refugee camps, including those in

Yemen and Bangladesh (World Health Organization (WHO), 2017a). Of these, a Rohingya

51 refugee camp in Bangladesh, which is temporarily located in Cox's Bazar, experienced a large-

52 scale diphtheria epidemic. As of 26 December 2017, the cumulative number of 2,526 cases and

27 deaths were reported (WHO, 2017a). To interrupt chains of transmission, emergency

vaccination has been conducted among children since 12 December 2017, achieving the overall coverage greater than $90 \%$ by the end of 2017 (WHO, 2018). Due to vaccination effort and other countermeasures, including contact tracing and hospital admission of cases, the epidemic has been brought under control, with incidence beginning to decline by the end of December 2017 (WHO, 2017a).

Considering that diphtheria has become a rare disease in industrialized countries, epidemiological information on model parameters that govern the transmission dynamics has

61 become very limited, and thus, it is valuable to assess how transmissible diphtheria would be 
62 through the analysis of the recent outbreak data. The basic reproduction number, $R_{0}$, is

63 interpreted as the average number of secondary cases that are produced by a single primary case

64 in a fully susceptible population, acting as the critical measure of the transmissibility. To date, an

65 explicit epidemiological estimate of $R_{0}$ for diphtheria has been reported only by Anderson and

66 May (1982): using a static modeling approach to age-dependent incidence data with an

67 assumption of the endemic equilibrium, $R_{0}$ was estimated as 6.6 in Pennsylvania, 1910s and 6.4

68 in Virginia and New York from 1934-47. Subsequently, a few additional modeling studies of

69 diphtheria took place (Kolibo, 2001; Sornbundit, 2017; Torrea, 2017), but none of these offered

70 an empirical estimate of $R_{0}$.

71 Here we analyze the epidemiological dataset of diphtheria in Rohingya refugee camp,

72 2017, aiming to estimate $R_{0}$ in this particular epidemic setting. Given that the epidemic occurred

73 among refugees, we explicitly account for uncertainties associated with unknown background

74 information, including the fraction of previously immune individuals and ascertainment rate of 75 cases.

\section{Materials \& Methods}

\section{Epidemiological data}

78 The latest epidemic curve was extracted from the report of the World Health Organization

79 (WHO) Regional Office for South East Asia (SEARO) (WHO, 2017a). Figure 1 shows the latest

80 available epidemic curve. As of 26 December 2017 (the latest date of observation), a total of

812,526 cases have been reported. Cases consist of (i) confirmed cases: cases reported as positive

82 for C. diphtheriae by multiplex assay, (ii) probable cases: cases with upper respiratory tract

83 illness with laryngitis or nasopharyngitis or tonsillitis AND sore throat or difficulty swallowing 
84 and an adherent membrane (pseudomembrane) OR gross cervical lymphadenopathy, and (iii)

85 suspected cases: any case with a clinical suspicion of diphtheria, including cases that are

86 unclassified due to missing values (WHO, 2017b). Prior to 11 December 2017, cases satisfied

87 only the condition of suspected cases. The definition was improved on and after 12 December

88 (WHO, 2017c), enforcing to count mainly probable cases, while not restricting the reporting of

89 suspected cases. For this reason, cases reported by 11 December are considered to have been

90 perhaps over-ascertained compared with cases that were reported later under improved case

91 definition. Mass child vaccination started on 12 December, and according to the administrative

92 coverage, greater than 90\% vaccination coverage was achieved by 30 December (WHO, 2018).

93 Vaccine-induced immunity requires at least 7-14 days to become effective, and moreover, the

94 reporting delay was assumed to be about 4 days (based on additional analyses; Results not

95 shown). For these reasons, the dataset from 23-26 December was discarded as the number of

96 cases may be influenced by emergency vaccination and also biased by the reporting delay.

\section{Modeling methods}

98 To formulate the epidemiological model, here we mathematically capture the epidemiological

99 process of secondary transmission using $R_{0}$ and the serial interval, i.e., the time from illness

100 onset in the primary case to illness onset in the secondary case. We assume that secondary

101 transmission does not take place before illness onset. According to a classical study by Stocks

102 (1930) in the United Kingdom (UK), the time interval from first to second diphtheria cases in the

103 household revealed a bimodal shape. Following Klinkenberg and Nishiura (2011), the first peak

104 corresponds to an independent infection in the community and the second peak reflects within-

105 household transmission. As one of peak time-lags in the observed time interval was observed on

106 day 8 , we assumed that the mean serial interval was 8 days, and we imposed an assumption that 
107 the coefficient of variation $(\mathrm{CV})$ of the serial interval distribution was $50 \%$, and later varied it

108 from $25 \%$ to $75 \%$ as part of the sensitivity analysis.

109 To capture the epidemiological phenomena of reproduction, it is very well known that the

110 renewal process can capture the serial stochastic dependence structure (Nishiura, 2010). Let $i_{t}$ be

111 the number of new cases on day $t . g_{\tau}$ represents the distribution of the serial interval. To describe

112 the time-dependent incidence $i_{t}$ on day $t$, we have

$$
i_{t}=R_{0} s_{t} \sum_{\tau=1}^{t-1} i_{t-\tau} g_{\tau}
$$

114 where $s_{t}$ represents the fraction of susceptible individuals on day $t$. The renewal process of this

115 type is not original to the present study and has been applied to other settings including real-time

116 epidemic modelling studies (Asai \& Nishiura, 2017; Dinh et al., 2016; Ejima \& Nishiura, 2018;

117 Endo \& Nishiura, 2015; Nishiura et al., 2010; Nishiura et al., 2016; Tsuzuki et al., 2017). It

118 should be noted that the incidence $i_{t}$ includes both symptomatic and asymptomatic cases. Let $c_{t}$

119 be the reported number of cases on calendar day $t$. Supposing that only the fraction $\alpha_{t}$ among the 120 total number of infections are diagnosed and reported, $c_{t}$ satisfies

$$
i_{t}=\frac{c_{t}}{\alpha_{t}}
$$

where $\alpha_{t}$ is modeled as a function of $t$. Because the case definition was improved from 12

123 December 2017 onward, the ascertainment rate likely varied around that time. Namely, we set $\alpha_{t}$

$124=a_{1}$ for time by 11 December and $a_{2}$ on 12 December and later. We assumed that only the

125 ascertainment rate changed as a function of time, and also that $R_{0}$ and depletion of susceptible

126 individuals were unaffected by time.

We model the fraction susceptible $s_{t}$ on day $t$ in the following way. Let $v$ represent the

128 previously immunized fraction so that only fraction $(1-v)$ of the population is susceptible at the 
129 beginning of the epidemic. In addition to the previously immune fraction, $s_{t}$ decreases when

130 natural infection takes place. Suppose that the total population size was $N, s_{t}$ is written as

$$
S_{t}=1-v-\frac{\sum_{y=1}^{t-1} \frac{c_{y}}{\alpha_{y}}}{N} \text {. }
$$

132 We assume that $N$ is equal to the population size of epidemic area within Rohingya refugee camp as 579,384 persons (Banerji \& Ahmed, 2017). Accordingly, the renewal equation is written as

$$
E\left(c_{t} ; R_{0}, v, a_{1}, a_{2}\right)=\alpha_{t} R_{0}\left(1-v-\frac{\sum_{y=1}^{t-1} \frac{c_{y}}{\alpha_{y}}}{N}\right) \sum_{\tau=1}^{t-1} \frac{c_{t-\tau}}{\alpha_{t-\tau}} g_{\tau}
$$

135 where $\tau$ in the right-hand side indicates the time since infection (or the so-called "infection-age").

136 We assume that $c_{t}$ follows a Poisson distribution. The likelihood to estimate $\theta$ consisting of the

137 parameters $R_{0}, v$ and $\alpha_{t}$ is derived as

$$
L\left(\theta ; \mathbf{c}_{\mathbf{T}}\right)=\prod_{t=1}^{T}\left(\frac{E\left(c_{t}\right)^{c_{t}} \exp \left(-E\left(c_{t}\right)\right)}{c_{t} !}\right)
$$

139 where $T$ is the latest time of observation (i.e., 22 December in our case study) and $\mathbf{c}_{\mathbf{T}}=\left(c_{1}, c_{2}, \ldots\right.$,

$\left.140 c_{T}\right)$

\section{Uncertainty and sensitivity analyses}

142 While we specified unknown parameters as $R_{0}, v$ and $\alpha_{t}$, it is expected that $R_{0}$ is correlated with

143 initially immune fraction $v$ and also $\alpha_{t}$. Thus, it is vital to quantify $R_{0}$ while accounting for the

144 uncertainty of other model parameters. In the present study, $v$ was most uncertain and thus

145 subject to uncertainty analysis, while $\alpha_{t}$ was estimated as a step function governed by two

146 parameters, $a_{1}$ and $a_{2}$. Uncertainty in parameter values can be addressed by randomly sampling 
147 the uncertain parameter value from probability distributions (Gilbert et al., 2014). Here we use

148 the Latin Hypercube sampling (LHS) method (Sanchez \& Blower, 1997) in which a symmetric

149 triangular distribution of $v$ was assumed to be in the range from 0.0 to 0.7 ; the health survey of

150 Rohingya population indicated that overall $30.8 \%$ of children had received no vaccinations

151 (Guzek et al, 2017), and the remaining 69.2\% receives at least a single immunization, which is

152 not necessarily against diphtheria but against a specific disease. We restrict our interest to

153 diphtheria, and thus, the vaccination coverage against diphtheria should be lower than that of

154 "any" vaccine. Thus, we expect that the actual coverage is nearby the mid-point of the range

155 from 0.0 to 0.7 . In addition, we examined the sensitivity of $R_{0}$ to variations in the length of the

156 serial interval.

\section{Ethical considerations}

158 The present study analyzed data that is publicly available. As such, the datasets used in our study

159 were de-identified and fully anonymized in advance, and the analysis of publicly available data

160 without identity information does not require ethical approval.

\section{Results}

162 Figure 2 shows univariate distributions of estimated parameters $R_{0}$ and $\alpha_{t}$ based on Latin 163 hypercube sampling $(n=1000)$ of parameter $v$ from 0 to 0.70 with the peak at $0.35 . a_{1}$ reflects

164 ascertainment by 11 December 2017, while $a_{2}$ shows the same on and after 12 December. $R_{0}$ 165 took the minimum and maximum estimates at 4.7 and 14.8, respectively, with the median 166 estimate at 7.2. The distribution was skewed to the right with the mode at 6.7. Excluding lower

167 and upper tails, 950 samples (95\%) of $R_{0}$ (or what it can be assumed as the $95 \%$ tolerance

168 intervals) were in the range of 5.0 to 12.3 . Distributions of $a_{1}$ and $a_{2}$ were also right skewed. $a_{1}$ 
169 ranged from 0.007 to 0.021 with the median 0.010 , while $\alpha_{2}$ ranged from 0.003 to 0.011 with the

170 median 0.005. Lower and upper 95\% tolerance intervals of $a_{1}$ and $a_{2}$ were $(0.007,0.017)$ and

171 (0.004, 0.009), respectively. A decline of $\alpha_{t}$ was observed, because the incidence was supposed

172 to have grown more during the early phase (i.e. by 11 December) given the estimated

173 epidemiological parameters. In practical sense, the timing coincided with the involvement of

174 contact tracing practice enforced by the Médecins Sans Frontières.

175 Figure 3 shows the distributions of two estimated parameters in two dimensional spaces

176 and also the comparison between observed and predicted epidemic curve. As can be expected

177 from equation (4), $R_{0}$ and $v$ were positively correlated given an epidemic curve. Specifically, as $v$

178 increases $R_{0}$ must also increase to achieve an identical epidemic curve. Similarly, $R_{0}$ was

179 positively correlated with ascertainment rates, $a_{1}$ and $a_{2}$. While the ascertainment might have

180 been lowered due to the stricter case definition, the small estimate of $a_{2}$ can also indicate that a

181 substantial fraction of undiagnosed individuals existed and the susceptible fraction was then

182 gradually depleted in the population. The observed and predicted epidemic curves are compared

183 in the right lower panel of Figure 3. While the model is kept simple with four unknown

184 parameters, the predicted epidemic curve overall captured the observed pattern.

185

Sensitivity of $R_{0}$ to the variation in the serial interval is shown in Figure 4 . We varied the

$186 \mathrm{CV}$ of the serial interval distribution from $25 \%$ to $75 \%$. When the $\mathrm{CV}$ was $25 \%$, the median and

187 mode of $R_{0}$ from Latin hypercube sampling were 9.4 and 8.2, respectively. When the CV was

$18875 \%$, the median and mode of $R_{0}$ were estimated to be 5.7 and 5.2, respectively. That is, as the

189 variance increases, the estimate of $R_{0}$ decreases. However, the variation in $R_{0}$ induced by the CV

190 was smaller than the uncertainty associated with the initially immune fraction.

191 


\section{Discussion}

193 The present study estimated $R_{0}$ of diphtheria in the Rohingya refugee camp, explicitly accounting

194 for case ascertainment and previously immune fraction. Since previously immune fraction $v$ of

195 the refugee population was not precisely known, uncertainty analysis of $R_{0}$ was conducted with

196 an input parameter assumption for $v$ employing the Latin Hypercube sampling method. $R_{0}$ ranged

197 from 4.7 to 14.8 with the median estimate at 7.2. To our knowledge, the present study is the first

198 to statistically estimate $R_{0}$ of diphtheria from epidemic data. For the statistical estimation of $R_{0}$,

199 the renewal process model was employed, which has an advantage to handle the right-censored

200 data during the course of an epidemic, compared with other available methods for completely

201 observed data, e.g., Nishiura (2010).

202 Estimated median $R_{0}$ was broadly consistent with the value ranging from 6 to 7 as

203 indicated by Anderson and May (1982) based on a static model for endemic data that uses the

204 age-dependent incidence in the UK. We have shown that the frequently quoted estimate agrees

205 well with dynamically estimated $R_{0}$ from the refugee camp in the present day. Assuming $R_{0}=7$, to

206 control diphtheria by means of mass vaccination, the coverage greater than $86 \%$ must be

207 satisfied. Since our study focused on uncertainty and sensitivity analyses, the exact estimate of

$208 R_{0}$ cannot be pointed out. However, despite the uncertainty regarding $v$ in this population, we

209 estimated a distribution of $R_{0}$ consistent with previous estimates. While the mode of distribution

210 for $R_{0}$ was 6.7 , the validity of this value depends on the validity of our prior distribution of $v$,

211 which was not supported by any published evidence of this refugee population. Nevertheless,

212 Demographic and Health Survey data of the Rohingya population in Myanmar indicated a close

213 value from $40-50 \%$ as the coverage of DTP (Ministry of Health and Sports, 2017). 
214 Ascertainment rates were jointly estimated only by using the epidemiological case data and the

215 population size.

216 What we have shown in the present study is that when we have an access to not only the

217 initial growth rate of the epidemic but also the incidence data around the time at which peak

218 incidence is observed, $R_{0}$ and susceptible fraction can potentially be jointly quantified. Even

219 without explicit estimate of the initially immune fraction, we have shown that an indication of

220 the possible value of $R_{0}$ can be obtained through uncertainty analysis. LHS appeared to be

221 particularly useful in the setting of refugee camp in which the background health status is not

222 well quantified (Helton \& Davis, 2002; Nishiura et al., 2017). LHS can offer probabilistic

223 distribution of the outcome measure, $R_{0}$ in our case, and this method appeared to be particularly

224 useful when one or more uncertain input information exist (Elderd et al., 2006; Coelho et al.,

225 2008; Samsuzzoha et al., 2013; Gilbert et al., 2014). While Bayesian modeling has replaced LHS

226 to some extent of uncertainty analysis as it can also offer posterior distributions of even uncertain

227 parameters (Elderd et al., 2006; Coelho et al., 2008), there could be an issue of identifiability

228 when two or more parameters are evidently correlated, e.g. as anticipated between $R_{0}$ and $v$ in

229 our model (4). In such an instance, we cannot be sure if the limited epidemic data with the

230 Bayesian estimation method can offer identifiable distributions for all parameters, and then LHS

231 can remain to act as a useful tool for uncertainty analysis.

232 Estimated small ascertainment rate during the epidemic is considered as reflecting the

233 time-dependent diagnostic practice at a local level. In fact, the small ascertainment rate can lead

234 us to observing the low case fatality risk of diphtheria in the Cox's Bazar that has been estimated

235 to be small $(27 / 2526=1 \%)$, although the right censoring would of course matter for the real time

236 interpretation (Nishiura et al., 2009; Mizumoto et al., 2015). Nevertheless, cases could have died 
237 in the community unnoticed with low specificity of the case definition, and this is endorsed as a 238 possible reason for observing the small number of deaths by the WHO (2017d). A follow-up

239 study in this regard should be conducted in the future.

240 Several limitations must be noted. First, our model rested on a homogeneous mixing

241 assumption. No heterogeneous patterns of transmission, including contact patterns and age-

242 dependency were taken into account due to shortage of information. In addition, the time-

243 dependent heterogeneity, including the reduced transmission potential due to contact tracing and

244 rapid hospitalization, was not explicitly taken into account due to insufficiency of the data. If

245 there were any additional indications or datasets that would allow explicit quantification of the

246 effective reproduction number from 12 December, that could give additional insights into the

247 success of control measures. Second, for similar reasons, no spatial information was explicitly

248 incorporated into the model. Third, a little more realistic features of refugee population, such as

249 the impact of migration on the epidemic were unfortunately discarded in the present study.

250 Similarly, one could investigate how overcrowding and malnutrition in the deprived population

251 would help enhance the spread of diphtheria, given sufficient data backup from epidemiological

252 investigations.

253 While these features need to be explicitly quantified in the future, we believe that our 254 study adds an important piece of evidence to the literature on diphtheria. The transmissibility of 255 diphtheria in the refugee population was estimated to be consistent with that in an endemic 256 setting and mass vaccination must satisfy at least the coverage of $86 \%$ to halt the major epidemic 257 of diphtheria. 


\section{Conclusions}

259 The present study estimated $R_{0}$ of diphtheria in the Rohingya refugee camp, explicitly accounting

260 for case ascertainment and previously immune fraction. Since previously immune fraction $v$ of

261 the refugee population was not precisely known, uncertainty analysis of $R_{0}$ was conducted with

262 an input parameter assumption for $v$ employing the Latin Hypercube sampling. $R_{0}$ ranged from

2634.7 to 14.8 with the median estimate at 7.2. LHS can offer probabilistic distribution of the

264 outcome measure, and this method appeared to be particularly useful in the setting of refugee

265 camp in which the background health status is poorly quantified.

\section{References}

267 Anderson RM, May RM. 1982. Directly transmitted infectious diseases: Control by

268 vaccination. Science 215(4536): 1053-1060.

269 Asai Y, Nishiura H. 2017. Joint estimation of the transmissibility and severity of Ebola virus

270 disease in real time. Journal of Biological Systems 25(4): 587-603.

271 Banerji A, Ahmed R. 2017. Rohingya 'rather die' than return to oppression in Myanmar.

272 Digital Journal, 15 December 2017. Available from:

273 http://www.digitaljournal.com/news/world/rohingya-rather-die-than-return-tooppression-in-myanmar/article/510061\#ixzz52RF2vNh9

Coelho FC, Codeço CT, Struchiner CJ. 2008. Complete treatment of uncertainties in a model for dengue R0 estimation. Cad Saude Publica. 24(4):853-61.

Dinh L, Chowell G, Mizumoto K, Nishiura H. 2016. Estimating the subcritical

279 transmissibility of the Zika outbreak in the State of Florida, USA, 2016. Theor Biol Med Model. 13(1):20. 
280 Elderd BD, Dukic VM, Dwyer G. 2006. Uncertainty in predictions of disease spread and

281 public health responses to bioterrorism and emerging diseases. Proc Natl Acad Sci U S A.

282

283

284

Guzek J, Siddiqui R, White K, van Leeuwen C, Onus R. 2017. Health survey in Kutupalong and Balukhali refugee settlements, Cox's Bazar, Bangladesh. Medecins Sans Frontieres: Belgium, 2017. Available from: https://www.msf.org/sites/msf.org/files/coxsbazar_healthsurveyreport_dec2017_final1.pd f

Helton JC, Davis FJ. 2002. Illustration of sampling-based methods for uncertainty and sensitivity analysis. Risk Anal. 22(3):591-622.

Hosseinpoor AR, Bergen N, Schlotheuber A, Gacic-Dobo M, Hansen PM, Senouci K, Boerma T, Barros AJ. 2016. State of inequality in diphtheria-tetanus-pertussis immunisation coverage in low-income and middle-income countries: a multicountry study of household health surveys. Lancet Glob Health 4(9):e617-26. 
303 Klinkenberg D, Nishiura H. 2011. The correlation between infectivity and incubation period

304 of measles, estimated from households with two cases. J Theor Biol 284: 52-60.

305 Kolibo DV, Romaniuk SI. 2001. Mathematical model of the infection process in diphtheria

306 for determining the therapeutic dose of antitoxic anti-diphtheria serum]. Ukr Biokhim Zh 307 73(2):144-51 (in Russian).

308 Ministry of Health and Sports, Myanmar. 2017. Myanmar Demographic and Health Survey 309 2015-16. Nay Pyi Taw, Myanmar \& The DHS Program, ICF, USA. 2017. Available 310 from: https://dhsprogram.com/pubs/pdf/FR324/FR324.pdf

311 Mizumoto K, Saitoh M, Chowell G, Miyamatsu Y, Nishiura H. 2015. Estimating the risk of 312 Middle East respiratory syndrome (MERS) death during the course of the outbreak in the 313 Republic of Korea, 2015. International Journal of Infectious Diseases 39:7-9. doi: $314 \quad$ 10.1016/j.ijid.2015.08.005.

315 Nishiura H, Klinkenberg D, Roberts M, Heesterbeek JA. 2009. Early epidemiological 316 assessment of the virulence of emerging infectious diseases: a case study of an influenza 317 pandemic. PLoS One 4(8):e6852. doi: 10.1371/journal.pone.0006852.

318 Nishiura H. 2010. Correcting the actual reproduction number: A simple method to estimate 319 R0 from early epidemic growth data. International Journal of Environmental Research $320 \quad$ and Public Health 7(1): 291-302.

321 Nishiura H, Chowell G, Safan M, Castillo-Chavez C. 2010. Pros and cons of estimating the 322 reproduction number from early epidemic growth rate of influenza A (H1N1) 2009. $323 \quad$ Theoretical Biology and Medical Modelling 7: 1 
324 Nishiura H, Kinoshita R, Mizumoto K, Yasuda Y, Nah K. 2016. Transmission potential of

325 Zika virus infection in the South Pacific. International Journal of Infectious Diseases.

$326 \quad 45: 95-7$.

327 Nishiura H, Tsuzuki S, Yuan B, Yamaguchi T, Asai Y. 2017. Transmission dynamics of

328 cholera in Yemen, 2017: a real time forecasting. Theor Biol Med Model 14:14.

329 Rusmil K, Gunardi H, Fadlyana E, Soedjatmiko, Dhamayanti M, Sekartini R, Satari HI,

330 Risan NA, Prasetio D, Tarigan R, Garheni R, Milanti M, Hadinegoro SR, Tanuwidjaja S,

331 Bachtiar NS, Sari RM. 2015. The immunogenicity, safety, and consistency of an

332 Indonesia combined DTP-HB-Hib vaccine in expanded program on immunization

333 schedule. BMC Pediatr 15:219.

334 Samsuzzoha M, Singh M, Lucy D. 2013. Uncertainty and sensitivity analysis of the basic

335 reproduction number of a vaccinated epidemic model of influenza. Appl Math Model.

$336 \quad 37(3): 903-915$.

337 Sanchez MA, Blower SM. 1997. Uncertainty and sensitivity analysis of the basic

338 reproductive rate, tuberculosis as an example. Am J Epidemiol. 145(12):1127-1137.

339 Sangal L, Joshi S, Anandan S, Balaji V, Johnson J, Satapathy A, Haldar P, Rayru R,

340 Ramamurthy S, Raghavan A, Bhatnagar P. 2017. Resurgence of Diphtheria in North

341 Kerala, India, 2016: Laboratory Supported Case-Based Surveillance Outcomes. Front

$342 \quad$ Public Health 5:218.

343 Sornbundit K, Triampo W, Modchang C. 2017. Mathematical modeling of diphtheria

344 transmission in Thailand. Comput Biol Med 87:162-168.

345 Stocks P. 1930. Infectiousness and immunity in regard to chickenpox, whooping-cough,

346 diphtheria, scarlet fever and measles. Proc R Soc Med 23:1349-68. 
347 Torrea M, Ortega D, Torera JL. 2017. A modeling of a Diphtheria epidemic in the refugees camps. bioRxiv Posted October 27, 2017. doi: https://doi.org/10.1101/208835 Dynamics of the pneumonic plague epidemic in Madagascar, August to October 2017. Eurosurveillance 22(46):pii=17-00710. Bazar, Bangladesh - Update \#2 (27 December 2017). World Health Organization: Geneva, 2017. Available from: https://reliefweb.int/sites/reliefweb.int/files/resources/bancxbdiphtheriaresponseupdate22 71217.pdf

World Health Organization (WHO). 2017b. Early Waning, Alert and Response System (EWARS). WHO: Geneva, 2017. Available from: http://www.who.int/emergencies/kits/ewars/en/ World Health Organization (WHO). 2017c. Minutes of Health Sector Meeting, 27 December 2017. WHO: Bangladesh, 2017. Available from: https://www.humanitarianresponse.info/system/files/documents/files/minutes_of_health_ sector_meeting_27_december_2017.pdf

World Health Organization (WHO). 2017d. Mortality and Morbidity Weekly Bulletin. Cox's Bazar, Bangladesh, Volume No 9: Week 49, 2017. WHO: Bangladesh, 2017. Available from: http://www.searo.who.int/bangladesh/mmwbwk49171224.pdf?ua=1

World Health Organization (WHO). 2018. Weekly Situation Report \#9. WHO: Bangladesh, 368 2018. Available from: https://reliefweb.int/sites/reliefweb.int/files/resources/sitrep09.pdf 


\section{Figures}

371 Figure 1. Daily incidence of diphtheria cases in Rohingya refugee camp, 2017

372 Daily number of new cases as extracted from the latest open data (WHO, 2017a). The vertical

373 axis represents the total of confirmed, probable and suspected cases. By 11 December 2017, the

374 count represents suspected cases. On and after 12 December 2017, the case definition was

375 improved, and probable cases replaced the majority.

376 Figure 2. Estimated values of the basic reproduction number and case ascertainment

377 rate

378 Univariate probability distribution of the basic reproduction number, $a_{1}$ by 11 December and $a_{2}$

379 from 12 December from Latin Hypercube sampling $(n=1,000)$. During the Latin Hypercube

380 sampling, the vaccination coverage, $v$, has a symmetric triangular distribution ranging from 0.0

381 to 0.7 .

382 Figure 3. Estimated correlations in each pair of estimated parameters, and comparison

383 between observed and predicted epidemic curves

384 Three panels except for right lower panel represent two-dimensional plot of estimated

385 parameters. During the Latin Hypercube sampling $(n=1,000)$, the vaccination coverage, $v$, has a

386 symmetric triangular distribution ranging from 0.0 to 0.7 . Lower right panel is the comparison

387 between observed and predicted epidemic curves. Bars constituting the epidemic curve show the

388 observed data, while dots indicate predicted epidemic curve from Latin Hypercube sampling $(n=$ $3891,000)$. 
390 Figure 4. Sensitivity of $R_{0}$ with respect to the serial interval

$391 R_{0}$ was estimated with variable values of the coefficient of variations $(\mathrm{CV})$ of the serial interval.

392 Mean serial interval was fixed at 8 days. Variations of $R_{0}$ along the vertical axis reflects the

393 uncertainty associated with the initially immune fraction $v$ of the Rohingya refugee population.

394 
Figure 1

Daily incidence of diphtheria cases in Rohingya refugee camp, 2017

Daily number of new cases as extracted from the latest open data (WHO, 2017a). The vertical axis represents the total of confirmed, probable and suspected cases. By 11 December 2017, the count represents suspected cases. On and after 12 December 2017, the case definition was improved, and probable cases replaced the majority.

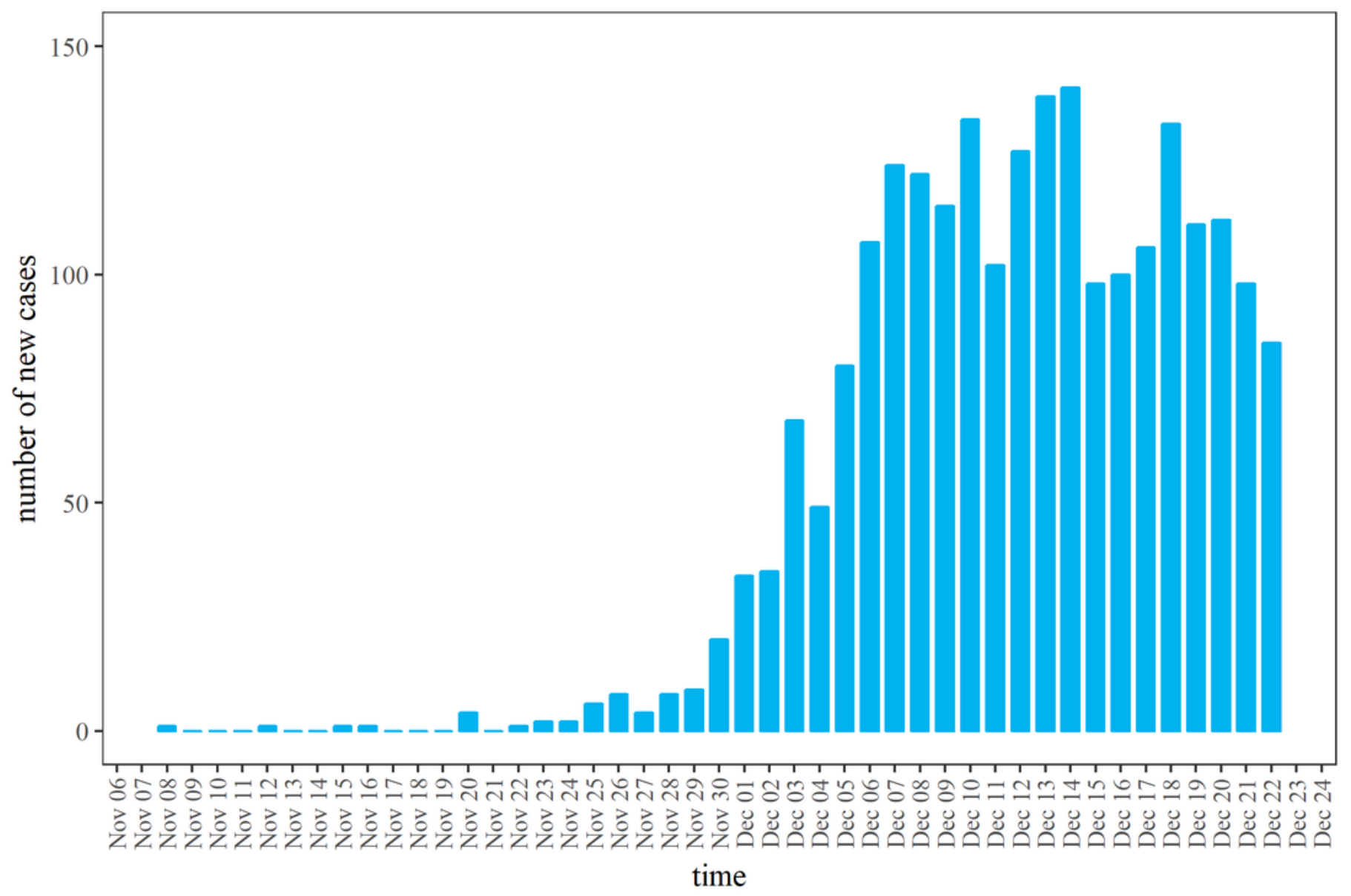




\section{Figure 2}

Estimated values of the basic reproduction number and case ascertainment rate Univariate probability distribution of the basic reproduction number, $a_{1}$ by 11 December and $a_{2}$ from 12 December from Latin Hypercube sampling $(n=1,000)$. During the Latin Hypercube sampling, the vaccination coverage, $v$, has a symmetric triangular distribution ranging from 0.0 to 0.7 .
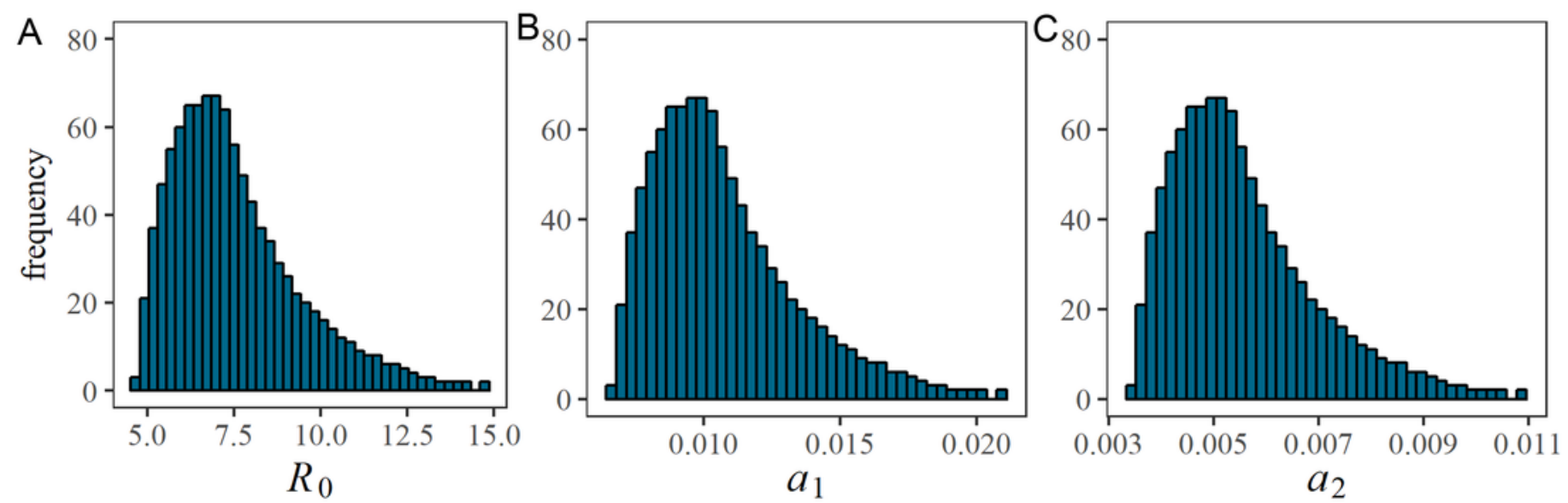
Figure 3

Estimated correlations in each pair of estimated parameters, and comparison between observed and predicted epidemic curves

Three panels except for right lower panel represent two-dimensional plot of estimated parameters. During the Latin Hypercube sampling $(n=1,000)$, the vaccination coverage, $v$, has a symmetric triangular distribution ranging from 0.0 to 0.7 . Lower right panel is the comparison between observed and predicted epidemic curves. Bars constituting the epidemic curve show the observed data, while dots indicate predicted epidemic curve from Latin Hypercube sampling $(n=1,000)$.
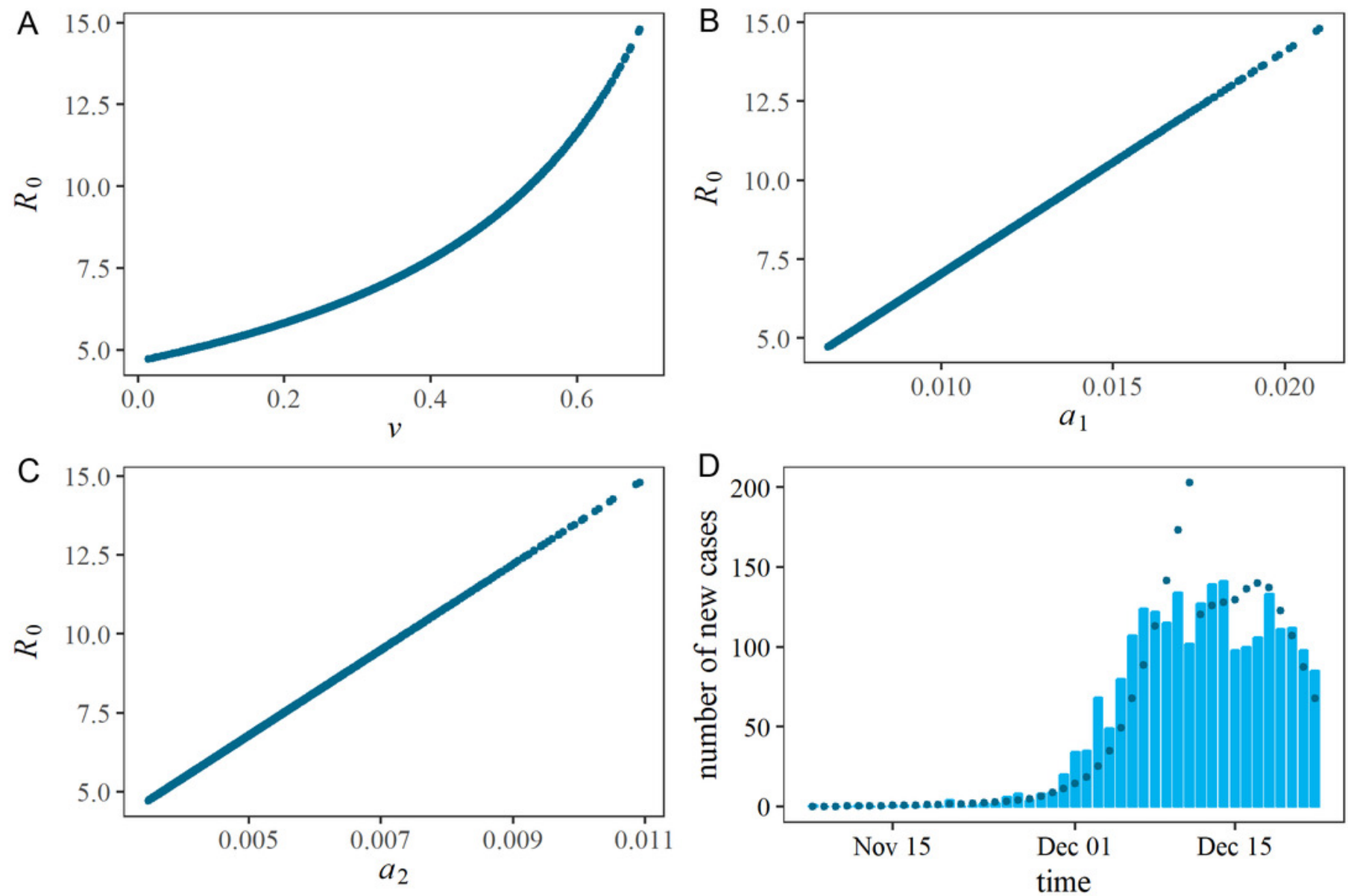


\section{Figure 4}

Sensitivity of $R_{0}$ with respect to the serial interval

$R_{0}$ was estimated with variable values of the coefficient of variations (CV) of the serial interval. Mean serial interval was fixed at 8 days. Variations of $R_{0}$ along the vertical axis reflects the uncertainty associated with the initially immune fraction $v$ of the Rohingya refugee population. 


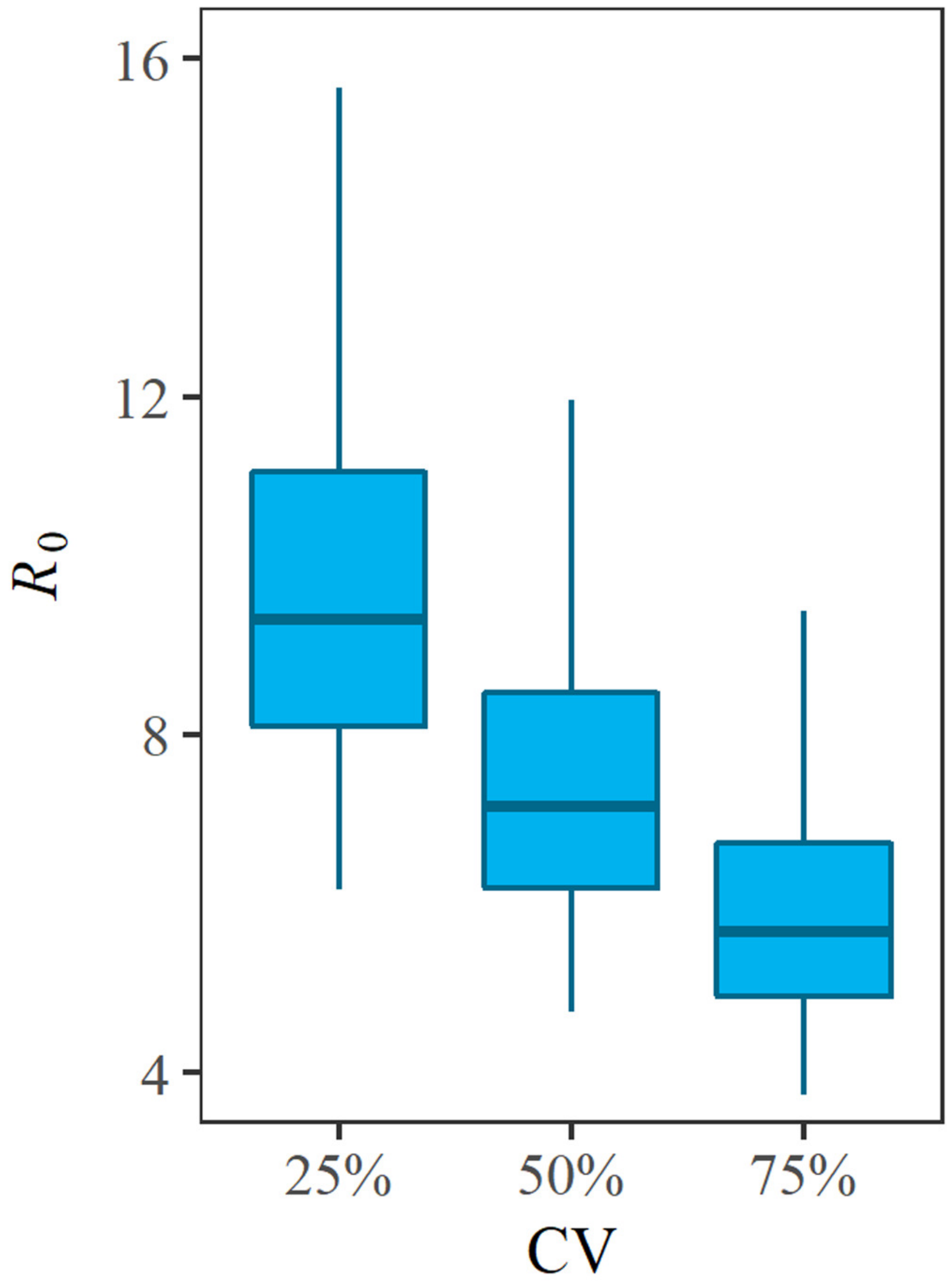

